\title{
Application of Simulation Technology in Transport Logistics Practice Teaching
}

\author{
Yang Han \\ Department of International Trade, Shanghai University of Engineering Science, Shanghai, China \\ hanyang2008@gmail.com
}

\begin{abstract}
Transport logistics courses require innovation in teaching methods, and application of simulation technology in transport logistics practices teaching is an effective way to meet this demand. In this paper, service model is used for modeling and simulation in the practice teaching of transport logistics to obtain operation results in the course of transport logistics, locate the transportation bottleneck and find the relevant optimization indicators in transportation. Thus it helps students to improve abilities to operate, respond, and collaborate.
\end{abstract}

Keywords—Simulation, service model, Transport Logistics, Practice Teaching

\section{仿真技术在运输物流实践教学中的应用}

韩杨

上海工程技术大学贸易经济系，上海，中国

摘 要 运输物流课程的实践性要求教学方法的创新, 将仿真技术应用于运输物流的实践教学是一种有效的手段。本文通过在运输 物流的实践教学环节应用 service model 仿真软件进行运输物流的建模与仿真, 得到运输过程中各物流节点的运行效果, 发现运输全程 的瓶颈所在，并得出运输过程的相关优化指标，最终在教学中达到提高学生操作能力、应变能力、协同能力的目标。

关键词 仿真, service model 软件, 运输物流, 实践教学

\section{1. 引言}

随着物流在我国的快速发展, 物流专业教育也得到了 迅速发展。物流专业具有很强的实践性和实用性。因此, 将高校的物流专业教育与实践教育紧密结合, 具有非常重 要的意义。在各高校的物流专业课程体系的设置中, 运输 物流的课程大都居于核心地位, 但针对运输物流的实践教 学研究, 目前还处于初步阶段。

\section{2. 物流专业实践教学中存在的问题}

国内高校物流类专业实践教学的模式一般来说主要包 括校内实践、实习、课程设计、实践讲座四个方面[1]。其 中, 学生到企业的实习, 如果学校与企业间没有完善的协 同机制, 学生到企业中没有具体岗位, 无法实际参与业务 操作, 将很难实现课堂理论知识与实践的有机结合。校内

《运输物流模拟实习》实践教学建设项目支持 (项目编号: A-2600-12-01049)
实践的教学可以在校内的模拟实验室进行, 将有利于提高 学生的综合能力和专业水平。但实际应用中, 这种模式业 务流程相对简单, 实验实习功能单一, 真实性、综合性差, 很难满足专业教学和科研工作的实际需要。

\section{3. 物流仿真的作用}

仿真技术是一门以控制论、系统论、相似原理和信息 技术为基础的综合性技术，它以计算机和专用设备为工具， 利用系统模型对实际的或设想的系统进行动态试验。将仿 真技术与物流活动相结合的物流仿真针对物流系统进行系 统建模, 在计算机上编制相应应用程序, 模拟实际物流系 统运行状况, 并统计和分析模拟结果, 用以指导实际物流 系统的规划设计与运作管理。这种虚拟模型的建立可以测 试各种想法和假设条件, 快速对各种设想进行模拟验证, 找到最优解决方案。 


\section{4.仿真技术在运输物流研究与教学中应用的文献综述}

\section{1 仿真技术在运输物流领域应用的相关研究}

将仿真技术应用于运输物流的研究已经有一定的基 础。在国外, Jörn Schönberger 与 Herbert Kopfe[2]2012 年研 究了未来不确定的数量变化对以公路运输为基础的货运系 统收益管理的影响。为了提高对未来货运量变化信息的准 确预测, 提出了一个决策支持系统, 并使用计算机仿真来 评价该系统的实用性和有效性。Øyvind Berle 等[3]在 2013 年使用一个简化的仿真模型对液化石油气的海路运输进行 定量分析。Alan Harrison 和 Johannes Fichtinger[4]在 2013 年研究全球海运网络的关联性, 及通过仿真模拟每天、每 周海运数量的多变性对船方库存管理的影响。在我国, 罗 首章等[5]2003 年利用 Petri 网建立对于宝钢铁水运输系统 各个实体的仿真模拟, 通过不同方案下仿真结果的比较, 得出运输调度方式的优化方案。祁明明等[6]2010 年采用 eM-Plant 物流仿真软件建立钢铁厂铁水铁路运输模型, 突 破了传统铁路运输设备能力的计算思路, 将运输过程中的 动态因素和概率事件综合考虑到仿真模型之中。李金等 [7]2011 年将 Agent 智能化仿真技术应用于井下运输仿真模 型, 对井下运输进行较为全面、真实的过程仿真。以上的 研究都充分体现了仿真技术在运输物流中应用的广泛性。

\section{2 仿真技术在物流教学中应用的相关研究}

将仿真与物流教学活动相结合在我国也有一定的研究 基础。金贵林, 邵正宇 [8]2011 年针对物流教学的特点, 指 出系统建模与仿真可用应用于物流教学中的实践性课程设 计。刘宏宇, 刘永悦[9]2012 年根据 flexsim 仿真软件的特点, 构建了基于 flexsim 的仿真实验方案, 通过不同类型、不同 内容的实验教学过程, 建立了适合物流管理实践能力组成结 构的实验教学与考评方案。

根据以上研究分析, 国内对于物流教学领域的仿真应 用已经有一定的研究基础, 但针对运输教学领域的仿真软 件应用研究的不多。本文通过在教学中应用 service model 软件, 指导学生模拟运输过程, 通过学生对于建模过程的 掌握, 实现模型的运行, 并对运行结果进行数据分析, 使 得学生能通过实习课程中的仿真模拟来了解实际运输过程 中可能面对的问题, 并能针对运输瓶颈进行原因分析, 提 出优化方案和解决对策。

\section{5. 以 service model 为例的运输物流仿真实习}

\section{1 运输模型描述}

该模型主要针对制造型企业的产品运输过程。一个制
造商 (Supplier1) 生产工件 (Part), 使用钢板 (Metal) 来 制造; 另一个制造商 (Supplier2) 生产包装盒 (Box), 使 用纸板 (Paper) 来制造。当工件和纸盒被生产出来后, 这 些货物分别在两个制造商的装货场 (Supplier1_Dock, Supplier2_Dock) 通过柴油车 (Diesel) 被运载到物流中心 的仓库 (Warehouse) 中。这个仓库存储这些物料, 并向有 需要的企业来提供物料。模型的布局图如图 1 所示:

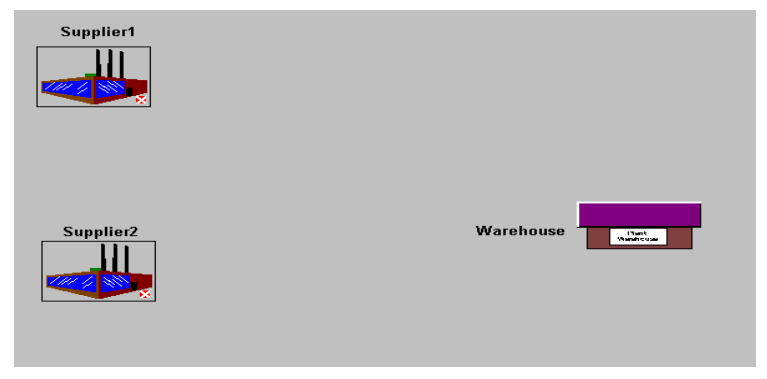

图 1 模型布局图

Metal 以每次 2 个到达 Supplier1。首次到达的时间为模 型运行时间 30 分钟, 以均值为 25 分钟的指数分布不间断 到达。Metal 在 Supplier1 加工成 Part 所需时间服从 $\mathrm{T}(3,8$, 12)。Paper 以每次 1 个到达 Supplier2, 首次到达时间为模 型开始运行时, 以均值为 15 分钟的指数分布, 共到达 8 个 批次。另一种类型的 Paper 以每次 1 个到达 Supplier2, 首 次到达时间为模型运行时间 150 分钟, 以均值为 30 分钟的 指数分布不间断到达。当 Paper 到达 Supplier2 后, 被加工 成 Box, Paper 在 Supplier2 加工成 Box 所需时间服从 T( 50, 64, 90)。

Part 需要 0.5 分钟到达 Supplier1_Dock。在 Supplier1_Dock, 20 个 Part 在一起装上 Diesel。Diesel 将 Part 运输到 Warehouse 并卸下。Diesel 运送 Part 从 Supplier1_Dock 到 Warehouse 的时间为 $75+/-12$ 分钟。然 后该 Diesel 返回到 Supplier1_Dock, 从 Warehouse 返回 Supplier1_Dock 的时间为 $55+/-8$ 分钟。Box 需要 0.5 分钟 到达 Supplier2_Dock。在 Supplier2_Dock, 每个 Box 单独装 上 Diesel。Diesel 将 Part 运输到 Warehouse 并卸下。Diesel 运送 Box 从 Supplier2_Dock 到 Warehouse 的时间为 75+/ -4 分钟, 从 Warehouse 返回 Supplier2_Dock 的时间为 75 $+/-4$ 分钟。

\section{2 利用 service model 建模及运行结果分析}

建模活动是通过对实际系统的观测和检测, 在忽略次 要因素及不可检测变量的基础上, 用物理或数学的方法进 行系统描述, 从而获得实际系统的简化近似模型[10]。本例 中的模型描述即是用 service model 对一项运输活动进行物 
理描述, 得到简化的近似模型。在此过程中, 用 build 命令 进行模型的建立, 其中的 location 模块定义运输节点, entity 模块定义所运送的货物, arrival 模块定义货物的出现规律, processing 模块定义货物的操作及运送逻辑。根据运输的实 际过程, 并考虑模型运行情况, 将运行时间定为 36 小时。

整个建模过程的逻辑流程如图 2 所示:

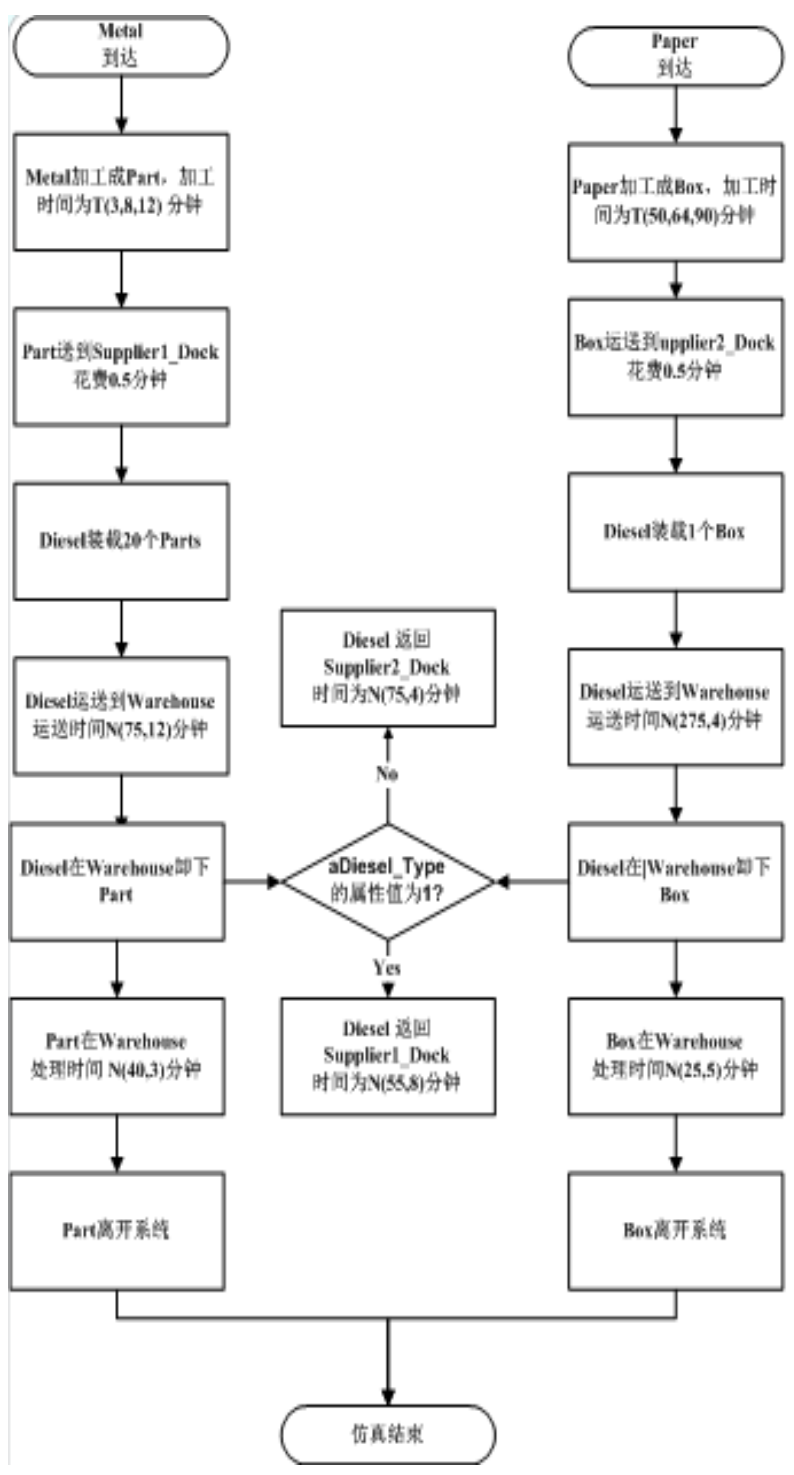

图 2 模型流程图
根据对该运输过程的模拟仿真, 得到运输过程各环节 的仿真现状。具体统计数据如表 1、2、3、4 所示:

由表 1 和表 3 中的数据可以看出, 生产纸盒的制造商 (Supplier2) 和生产工件制造商的货场（Supplier1_Dock） 货物停留的时间长, 始终处于忙碌状态, 没有空置的时间, 这说明纸盒制造商应该提高生产能力, 而工件制造商则要 提高货场的装卸货效率。

由表 2 和表 4 中的数据可以看出, 工件（metal）在整 个运输过程中等待的时间较长, 而纸盒 (box) 则是造成整 个运输过程瓶颈的重要因素。

表 1 运输过程中各物流节点相关统计数据

\begin{tabular}{l|r|r|r|r|r|}
\hline Name & Scheduled Time (HR) & \% Empty & \% Part Occupied & \% Full & \% Down \\
\hline Supplier1 & 36.00 & 67.93 & 32.07 & 0.00 & 0.00 \\
Supplier2 & 36.00 & 0.00 & 100.00 & 0.00 & 0.00 \\
Supplier1 Dock & 36.00 & 0.00 & 100.00 & 0.00 & 0.00 \\
Supplier2 Dock & 36.00 & 83.19 & 16.81 & 0.00 & 0.00 \\
Warehouse & 36.00 & 41.43 & 58.57 & 0.00 & 0.00 \\
\hline
\end{tabular}

表 2 货物及车辆的相关统计数据

\begin{tabular}{|l|r|r|r|r|}
\hline Name & \% In Move Logic & \% waiting & \% In Operation & \% Blocked \\
\hline Metal & 0.00 & 0.00 & 0.00 & 0.00 \\
\hline Part & 33.66 & 44.25 & 22.08 & 0.00 \\
\hline Paper & 0.00 & 0.00 & 0.00 & 0.00 \\
\hline Box & 28.66 & 0.00 & 36.35 & 34.98 \\
\hline DieselA & 0.00 & 0.00 & 0.00 & 0.00 \\
\hline
\end{tabular}

\section{6. 结论}

综上所述，利用仿真技术建立运输物流的仿真模型可 以使运输物流的各个环节在仿真平台中可视化显示。对仿 真模型的输出数据进行分析, 可找出运输过程中的瓶颈, 并使学生具备运用所学知识进行运输模拟的综合应用能 力。在此过程中, 通过学生间分组建模, 讨论模型运行结 果, 还可提高学生的协作能力。因此, 高校在物流专业教 学课程设置中可根据课程需要开设相关的物流仿真实验课 程, 让学生可以充分利用计算机仿真软件来练习, 增强学 生的实践能力。

表 3 运输过程中各物流节点的基本信息数据

\begin{tabular}{l|r|r|r|r|r|r|r|} 
Name & Scheduled Time (HR) & Capacity & Total Entries & Avg Time Per Entry (MIN) & Avg Contents & Maximum Contents & Current Contents \\
\hline Supplier1 & 36.00 & 999999.00 & 188.00 & 7.96 & 0.69 & 6.00 & 0.00 \\
Supplier2 & 36.00 & 999999.00 & 85.00 & 170.12 & 6.69 & 17.00 & 15.00 \\
Supplier1 Dock & 36.00 & 999999.00 & 12.00 & 625.21 & 3.47 & 4.00 & 3.00 \\
Supplier2 Dock & 36.00 & 999999.00 & 70.00 & 10.71 & 0.35 & 5.00 & 0.00 \\
Warehouse & 36.00 & 999999.00 & 76.00 & 26.46 & 0.93 & 5.00 & 2.00
\end{tabular}




\section{表 4 货物及车辆的移动轨迹数据}

\begin{tabular}{|c|c|c|c|c|c|c|c|}
\hline Name & Total Exits & Current Qty In System & Avg Time In System (MIN) & Avg Time In Hove Logic (MIN) & Avg Time Waiting (MIN) & Avg Time $\ln$ Operation (MIN) & Avg Time Blocked (MIN) \\
\hline Metal & 0.00 & 0.00 & 0.00 & 0.00 & 0.00 & 0.00 & 0.00 \\
\hline Part & 160.00 & 28.00 & 215.44 & 72.52 & 95.33 & 47.58 & 0.00 \\
\hline Paper & 0.00 & 15.00 & 0.00 & 0.00 & 0.00 & 0.00 & 0.00 \\
\hline Box & 66.00 & 4.00 & 261.65 & 75.00 & 0.00 & 95.12 & 91.53 \\
\hline DieselA & 0.00 & 9.00 & 0.00 & 0.00 & 0.00 & 0.00 & 0.00 \\
\hline
\end{tabular}

\section{参考文献(References)}

[1] M.-J. Wang, "Discussion on the Construction of Teaching System of University Logistics Practice," Logistics Engineering and Management, vol.35, no.229, pp.193-194, July 2013.

[2] Jörn Schönberger, Herbert Kopfer, "Revenue management in road-based freight transportation: Impacts of uncertainty of capacity consumption," Physical Distribution \& Logistics Management, Vol. 42, Iss: 4, pp.388-403, 2012.

[3] Øyvind Berle, Inge Norstad, Bjorn E. Asbjørnslett. "Optimization, risk assessment and resilience in LNG transportation systems," Supply Chain Management, Vol. 18, Iss: 3, pp.253-264, 2013.

[4] Alan Harrison, Johannes Fichtinger, "Managing variability in ocean shipping," Logistics Management, Vol. 24, Iss: 1, pp.7-21, 2013.

[5] S.-Z. Luo, F. Liu, M. zhou, and R.-Y. zhou, "The Application of Petri Net in the Iron Melt Transport Simulating System of Baosteel, "Journal of System simulation, vol.15, no.12, pp.1803-1804,1817,December 2003.
[6] M.-M. Qi, L. Wang, C. Yuan, and W. Gao, "eM-Plant Based Simulation Model for Hot Metal Transportation in Steel Plant," Industrial Engineering Journal, vol.13,no.2,pp. 28-33, April 2010

[7] X. Li, Z.-X. Li, and C.-P. Li, "Research on Underground Transportation Simulation Model Based on Agent Technology," Metal Mine, no.426, pp. 113-116,126, December 2011

[8] G.-L. Jin and Z.-Y. Shao, "The Application of System Modeling and Simulation to Logistics Teaching," Logistics Engineering and Management, vol.33, no.210, pp. 133-134, December 2011.

[9] H.-Y. Liu and Y.-Y. Liu, "An Explore Based on the Practical Ability for the Experiment Course Construction of Logistics Management Simulation Software," Logistics Engineering and Management, vol.34, no.213, pp. 210-211,225, March 2012.

[10] C.-X. Zhang,S.-Q. Li and L. Cai, “An Approach to Complex Engineering Modeling and Simulation," Systems Engineering, vol.25, no.2, pp. 111-115, February 2007. 